\title{
A Heuristic Approach for Minimum Set Cover Problem
}

\author{
Fatema Akhter \\ IEEE student member \\ Department of Computer Science and Engineering \\ Jatiya Kabi Kazi Nazrul Islam University Trishal, \\ Mymensingh-2220, Bangladesh
}

\begin{abstract}
The Minimum Set Cover Problem has many practical applications in various research areas. This problem belongs to the class of NP-hard theoretical problems. Several approximation algorithms have been proposed to find approximate solutions to this problem and research is still going on to optimize the solution. This paper studies the existing algorithms of minimum set cover problem and proposes a heuristic approach to solve the problem using modified hill climbing algorithm. The effectiveness of the approach is tested on set cover problem instances from ORLibrary. The experimental results show the effectiveness of our proposed approach.
\end{abstract}

Keywords-Set Cover; Greedy Algorithm; LP Rounding Algorithm; Hill Climbing Method

\section{INTRODUCTION}

For a given set system on a universe of items and a collection of a set of items, Minimum Set Cover Problem (MSCP) [1] finds the minimum number of sets that covers the whole universe. This is a NP hard problem proven by Karp in [2]. The optimization has numerous applications in different areas of studies and industrial applications [3]. The applications include multiple sequence alignments for computational biochemistry, manufacturing, network security, service planning and location problems [4]-[7].

Several heuristics and approximation algorithms have been proposed in solving the MSCP [8]. Guanghui Lan et al. proposed a Meta-RaPS (Meta-heuristic for Randomized Priority Search) [9]. Fabrizio Grandoni et al. proposed an algorithm based on the interleaving of standard greedy algorithm that selects the min-cost set which covers at least one uncovered element [10]. Amol Deshpande et al. [11] proposed an Adaptive Dual Greedy which is a generalization of Hochbaums [12] primal-dual algorithm for the classical Set Cover Problem.

This paper studies some popular existing algorithms of MSCP and proposes a heuristic approach to solve MSCP using modified hill climbing method. Within our knowledge, the same approach for MSCP of this paper has not been yet reported. Although this work implements two popular algorithms, Greedy Minimum Set Cover [14] and Linear Polynomial Rounding (LP) algorithm [15] to find solutions to MSCP, this work does not focus on the strength and weakness of the algorithms. The proposed approach starts with an initial solution from Greedy approach and LP rounding and then the result is optimized using modified hill climbing technique. The computational results shows the effectiveness of the proposed approach.

The rest of the paper is organized as follows: Section II describes the preliminary studies for proposed approach. Section III describes the proposed algorithm for MSCP. Section IV presents the experimental results. Section V provides the conclusion and future work.

\section{BACKGROUND THEORY AND STUDY}

This section briefly describes MSCP and presents some preliminary studies. This includes Greedy Algorithm, LP Rounding Algorithm, Hill Climbing Algorithm and OR Library of SCP instances.

\section{A. Minimum Set Cover Problem}

Given $a$ set of $n$ elements $U=\left[e_{1}, e_{2}, \ldots, e_{n}\right]$ and $a$ collection $S=\left\{S_{1}, S_{2}, \ldots, S_{m}\right\}$ of $m$ nonempty subsets of $U$ where $\bigcup_{i=1}^{m} S_{i}=U$. Every $S_{i}$ is associated with a positive cost $c\left(S_{i}\right) \geq 0$. The objective is to find a subset $X \subseteq S$ such that $\sum_{S_{i} \in X} c\left(S_{i}\right)$ is minimized with respect to $\bigcup_{S \in X} S=U$.

\section{B. Minimum k-Set Cover Problem}

An MSCP $(U, S, c)$ is a k-set cover problem [13] if, for some constant $k$, it holds that $\left|S_{i}\right| \leq k, \forall S_{i} \in S$ represented as $(U, S, c, k)$. For an optimization problem, $x^{O P T}$ presents an optimal solution of the problem where $O P T=f\left(x^{O P T}\right)$. For a feasible solution $x$, the ratio $\frac{f(x)}{O P T}$ is regarded as its approximation ratio. If the approximation ratio of a feasible solution is upper-bounded by some value $k$, that is $1 \leq \frac{f(x)}{O P T} \leq$ $k$, the solution is called an k-approximate solution.

\section{Greedy Minimum Set Cover Algorithm}

Data: Set system $(U, S), c: S \rightarrow Z+$

Input: Element set $U=[e 1, e 2, \ldots, e n]$, subset set $S=$ $\{S 1, S 2, \ldots, S m\}$ and cost function $c: S \rightarrow Z+$

Output: Set cover $\mathrm{X}$ with minimum cost 


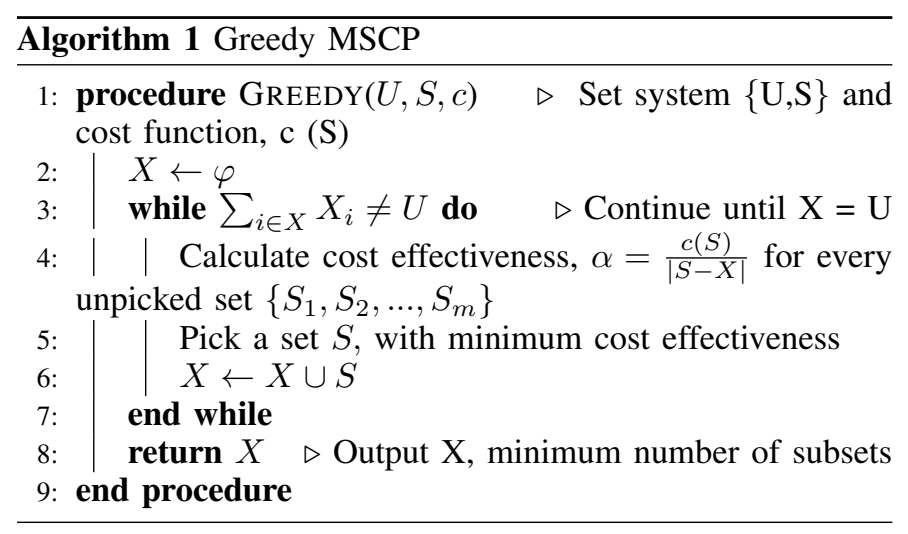

\section{LP Rounding Algorithm}

The LP formulation [15] of MSCP can be represented as

Minimize:

$$
\sum_{i=1}^{m} c_{i} \times X_{i}
$$

Subject To:

$$
\begin{array}{lr}
\sum_{i: e \in S_{i}} X_{i} \geq 1 & \forall e \in U \\
X_{i} \leq 1 & \forall e \in\{1,2, \ldots, m\} \\
X_{i} \geq 0 & \forall e \in\{1,2, \ldots, m\}
\end{array}
$$

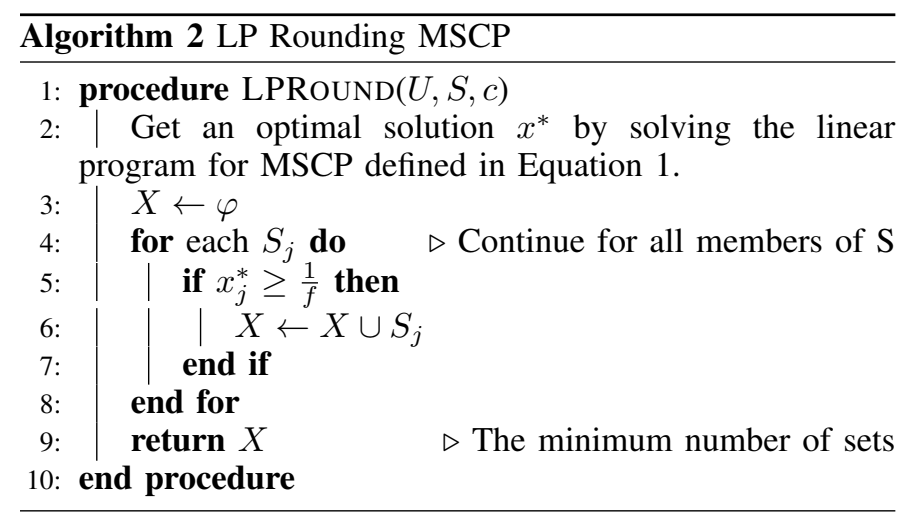

\section{E. Hill Climbing Algorithm}

Hill climbing [16] is a mathematical optimization technique which belongs to the family of local search. It is an iterative algorithm that starts with an arbitrary solution to a problem, then attempts to find a better solution by incrementally changing a single element of the solution. If the change produces a better solution, an incremental change is made to the new solution, repeating until no further improvements can be found.

\section{F. OR Library}

OR-Library [17] is a collection of test data sets for a variety of Operations Research (OR) problems. OR-Library was originally described in [17]. There are currently 87 data files for SCP. The format is

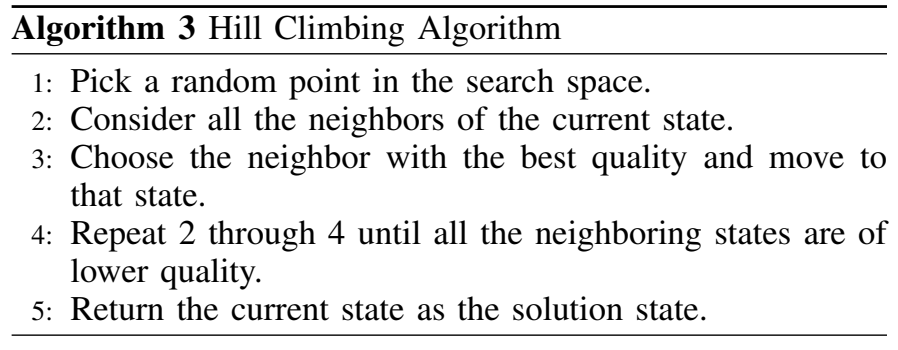

a) number of rows (m), number of columns $(n)$

b) the cost of each column $c(j), j=1,2, \ldots, n$

For each row $i(i=1, \ldots, m)$ : the number of columns which cover row $i$ followed by a list of the columns which cover row $i$.

\section{Proposed Algorithm}

This work modified the conventional hill climbing algorithm for set cover problem. To avoid the local maxima problem, this work introduced random re-initialization. For comparisons, greedy algorithm and LP rounding algorithm are used to find the initial state for the modified hill climbing algorithm. The evaluation function for the modified hill climbing algorithm is described below.

\section{A. Problem Formulation}

- Input: $N=|U|, U=[e 1, e 2, \ldots, e n], M=|S|, S=$ $\{S 1, S 2, \ldots, S m\}, c=\left\{c_{1}, c_{2}, \ldots, c_{m}\right\}$

- Output:

1) Minimum number of sets, $n(X)=|X|$.

2) List of minimum number of Sets, $X=$ $\left\{X_{1}, X_{2}, \ldots, X_{n(X)}\right\}$.

- Constraint: Universality of $X$ must hold, that is $\sum_{i \in X} X_{i}=U$.

- Objective:

1) Minimize the number of sets, $X$.

2) Minimize the total cost, $c(X)$.

\section{B. OR Library MSCP Formulation}

The formulation of MSCP for OR Library is given below.

1) Let $M^{m \times n}$ be a $0 / 1$ matrix, $\forall a_{i j} \in M_{i j}, a_{i j}=1$ if element $i$ is covered by set $j$ and $a_{i j}=0$ otherwise.

2) Let $X=\left\{x_{1}, x_{2}, \ldots, x_{n}\right\}$ where $x_{i}=1$ if set $i$ with cost $c_{i} \geq 0$ is part of the solution and $x_{i}=$ 0 otherwise.

Minimize:

$$
\sum_{i=1}^{n} x_{i} \times c\left(x_{i}\right)
$$

Subject To:

$$
\begin{array}{lr}
1 \leq \sum_{i=1}^{n} x_{i} \times a_{i j} & j \in\{1,2, \ldots, m\} \\
x_{i} \geq 0 & \forall x_{i} \in\{0,1\}
\end{array}
$$




\section{Proposed Algorithm}

This section describes our proposed algorithm for MSCP. The algorithm finds an initial solution and then optimizes the result using modified hill climbing algorithm.

\section{EXPERIMENTAL RESULTS AND DISCUSSIONS}

This section presents the computational results of the proposed approach. The effectiveness of the proposed approach is tested on 20 SCP test instances obtained from Beasley's OR Library. These instances are divided into 11 sets as in Table I, in which Density is the percentage of nonzero entries in the SCP matrix. All of these test instances are publicly available via electronic mail from OR Library.

The approach presented in this paper is coded using $\mathrm{C}$ on an Intel laptop with speed of $2.13 \mathrm{GHz}$ and 2GB of RAM under Windows 7 using the codeblock,version-13.12 compiler. Note here that this study presented here did not apply any kind of preprocessing on the instance sets received from ORLibrary. This paper did not report the CPU times or running time of the algorithm as they vary machine to machine and compiler to compiler.

TABLE I: Test instance details

\begin{tabular}{|c|c|c|c|c|c|}
\hline $\begin{array}{c}\text { Problem } \\
\text { Set }\end{array}$ & $\begin{array}{c}\text { Number of } \\
\text { instances }\end{array}$ & $\begin{array}{c}\text { Number of } \\
\text { rows(m) }\end{array}$ & $\begin{array}{c}\text { Number of } \\
\text { columns(n) }\end{array}$ & $\begin{array}{c}\text { Range of } \\
\text { cost }\end{array}$ & $\begin{array}{c}\text { Density } \\
\%\end{array}$ \\
\hline \hline 4 & 10 & 200 & 1000 & $1-100$ & $2 \%$ \\
5 & 10 & 200 & 2000 & $1-100$ & $2 \%$ \\
6 & 5 & 200 & 1000 & $1-100$ & $5 \%$ \\
$\mathrm{~A}$ & 5 & 300 & 3000 & $1-100$ & $2 \%$ \\
$\mathrm{~B}$ & 5 & 300 & 3000 & $1-100$ & $5 \%$ \\
$\mathrm{C}$ & 5 & 400 & 4000 & $1-100$ & $2 \%$ \\
$\mathrm{D}$ & 5 & 400 & 4000 & $1-100$ & $5 \%$ \\
$\mathrm{NRE}$ & 5 & 500 & 5000 & $1-100$ & $10 \%$ \\
$\mathrm{NRF}$ & 5 & 500 & 5000 & $1-100$ & $20 \%$ \\
$\mathrm{NRG}$ & 5 & 1000 & 10000 & $1-100$ & $2 \%$ \\
$\mathrm{NRH}$ & 5 & 1000 & 10000 & $1-100$ & $5 \%$ \\
\hline
\end{tabular}

\section{A. Experimental Results of Weighted SCP}

Table II presents the experimental results for the proposed approach for weighted SCP instances. The first column represents the name of each instance. The optimal or best-known solution of each instance is given in the 2 nd column. The 3 rd and 4th column represent the solution found using greedy and LP rounding approach. The 5th and 6th column represent the solutions found in [5] and [7]. The last two columns contain the result found using proposed approach, started from greedy approach and LP rounding approach respectively.

\section{B. Experimental Results of Unweighted SCP}

Table III presents the experimental results of the proposed approach for unweighted SCP instances. This paper used the same 20 instances of weighted SCP and made them unweighted by replacing the weights to 1 on these instances. The first column represents the name of each instance. The optimal or best-known solution of each instance is given in the 2 nd column. The 3 rd and 4 th column represent the solution found using greedy and LP rounding approach. The 5th and 6th column represent the solutions found in [18] and [19]. The last two columns contain the result found using proposed approach, started from greedy approach and LP rounding algorithm respectively.

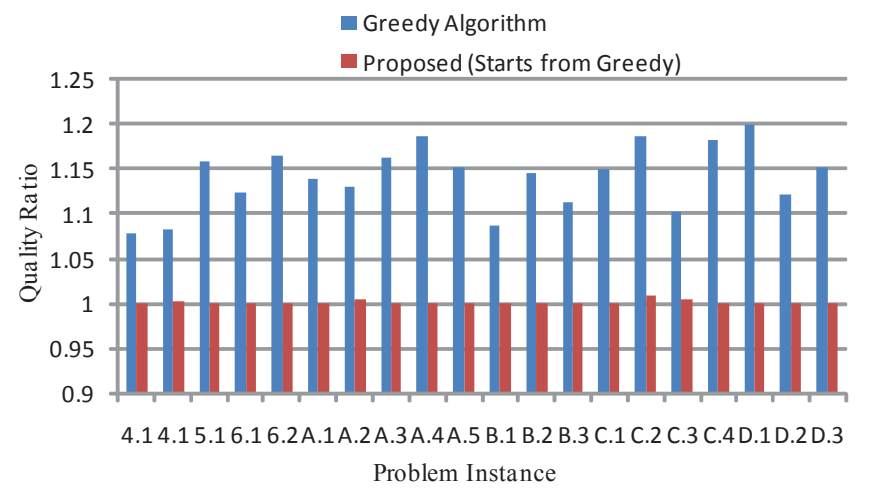

Fig. 1: Quality ratio of weighted problem instances for Greedy and Proposed Algorithm.

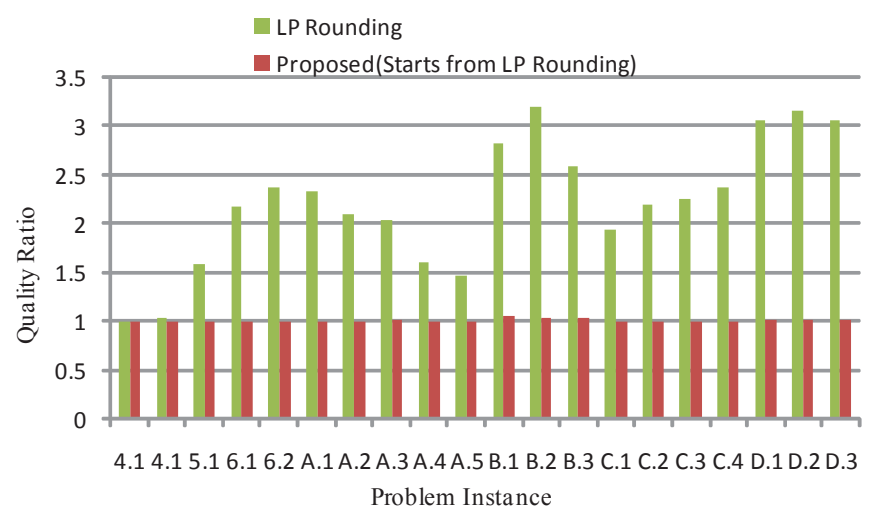

Fig. 2: Quality ratio of weighted problem instances for LP Rounding and Proposed Algorithm.

\section{Result Summary}

Summary: The optimal solution presented in Table II and Table III are taken from [7]. The quality of a solution derived by an algorithm is measured by Quality Ratio which is defined as a ratio of the derived solution to the optimal solution. The quality ratio for each instance for conventional greedy algorithm, LP rounding and Proposed algorithms, presented in this work are shown in Fig. 1, 2, 3 and 4. The figures show the ratio values, plotted as histogram for every instance, presented in this work.

$$
\text { Quality Measure Ratio }=\frac{\text { Derived Solution }}{\text { Optimal Solution }}
$$

Another popular quality measurement reported in literature is called GAP which is defined as the percentage of the deviation of a solution from the optimal solution or best known solution. The summarized results, in terms of average quality and average GAP, for weighted set covering instances are presented in Table IV. For unweighted set covering instances it is represneted in Table $\mathrm{V}$. 


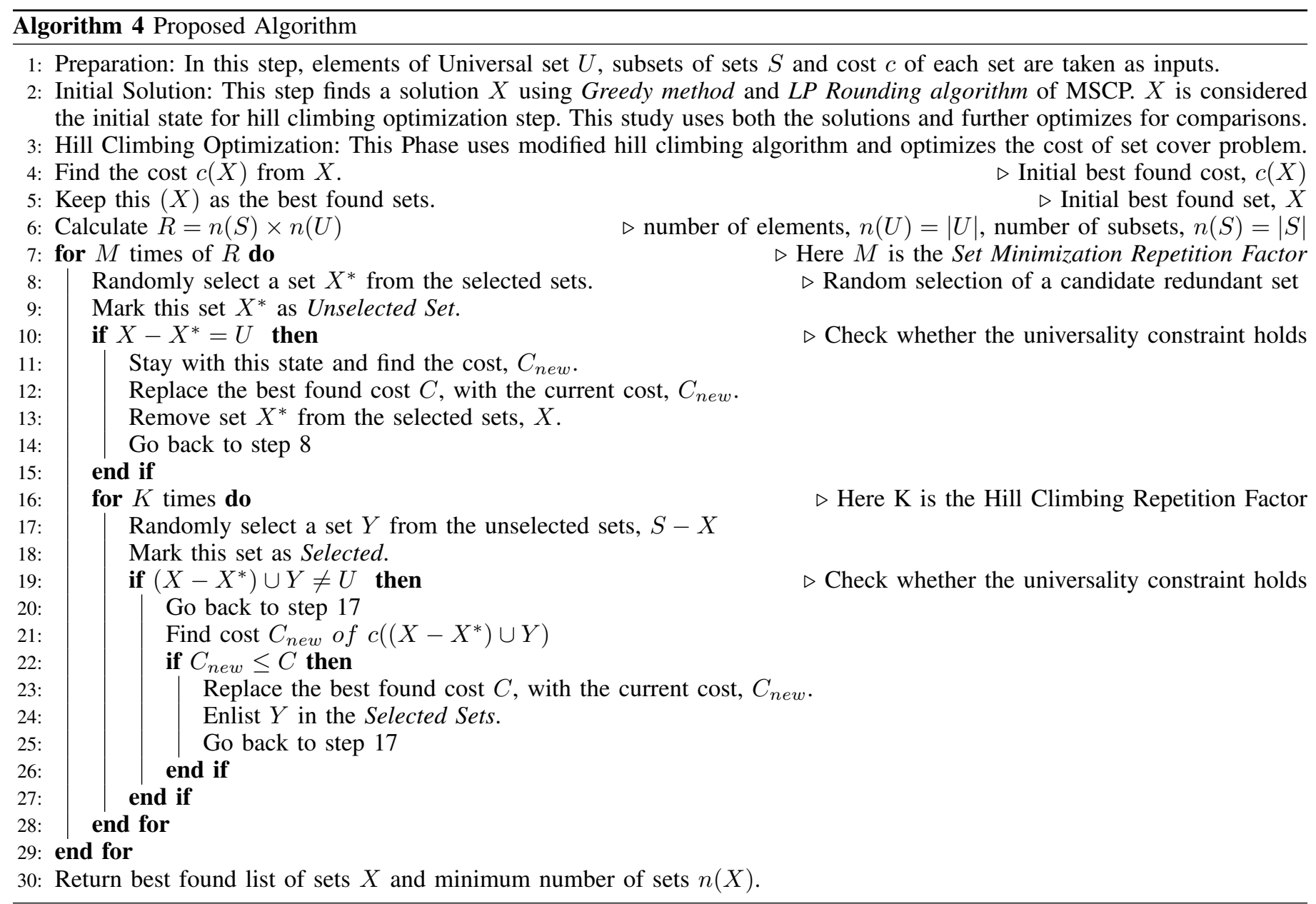

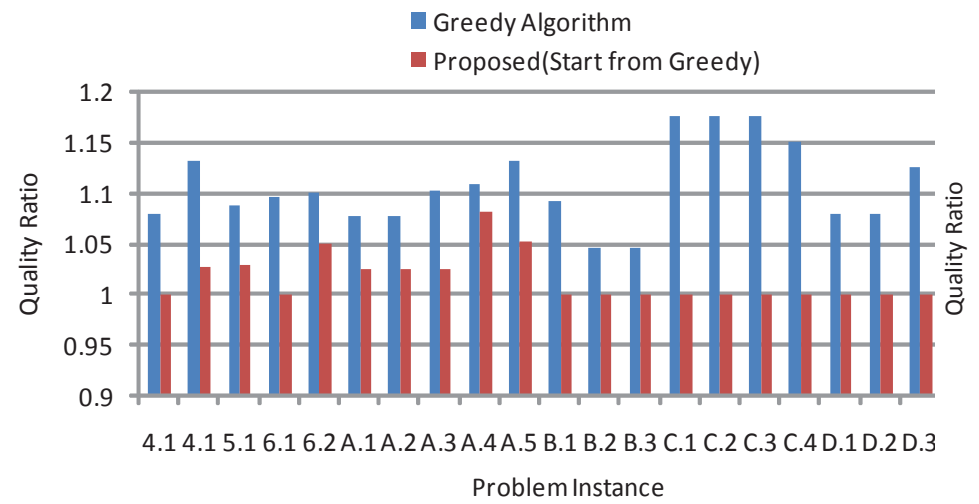

Fig. 3: Quality ratio of unweighted problem instances for Greedy and Proposed Algorithm.

$$
G A P=\frac{\text { Derived Solution }- \text { Optimal Solution }}{\text { Optimal Solution }} \times 100 \%
$$

The proposed algorithm presented in this paper used conventional greedy algorithm and LP-Rounding Algorithm as initial solution. Then with the modified hill climbing method,

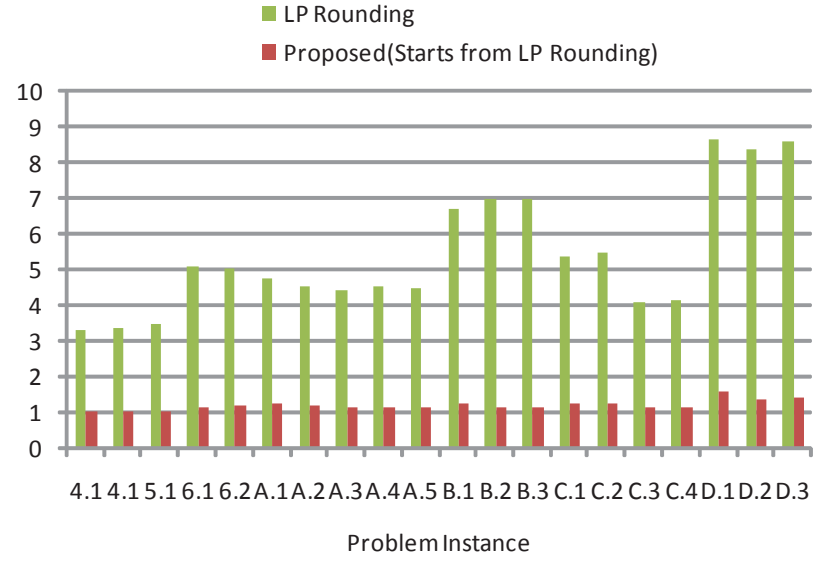

Fig. 4: Quality ratio of unweighted problem instances for LP Rounding and Proposed Algorithm.

these results are further optimized. Table IV and Table V compare the proposed heuristic approach to the original greedy approach and LP Rounding algorithm.

In Table IV, the average quality ratio and average GAP of original greedy are 1.14 and 14.10 respectively for weighted SCP while for proposed approach they are 1.00 and 0.09 . 
TABLE II: Experimental Results for Weighted SCP

\begin{tabular}{|c|c|c|c|c|c|c|c|}
\hline \multirow{2}{*}{$\begin{array}{l}\text { Instance } \\
\text { number }\end{array}$} & \multirow{2}{*}{$\begin{array}{l}\text { Optimal } \\
\text { Solution }\end{array}$} & \multirow{2}{*}{$\begin{array}{c}\text { Greedy } \\
\text { Algorithm }\end{array}$} & \multirow{2}{*}{$\begin{array}{c}\text { LP } \\
\text { Rounding }\end{array}$} & \multirow{2}{*}{$\begin{array}{c}{[5]} \\
\text { (Meta-RaPS) }\end{array}$} & \multirow{2}{*}{$\begin{array}{c}{[7]} \\
\text { (Descent Heuristic) }\end{array}$} & \multicolumn{2}{|c|}{ Proposed Algorithm } \\
\hline & & & & & & Start from Greedy & Start from LP Rounding \% \\
\hline 4.1 & 429 & 463 & 429 & 429 & 433 & 429 & 429 \\
\hline 4.10 & 514 & 556 & 539 & 514 & 519 & 515 & 514 \\
\hline 5.1 & 253 & 293 & 405 & 253 & 265 & 253 & 255 \\
\hline 6.1 & 138 & 155 & 301 & 138 & 149 & 138 & 138 \\
\hline 6.2 & 146 & 170 & 347 & 146 & 156 & 146 & 147 \\
\hline A. 1 & 253 & 288 & 592 & 253 & 258 & 253 & 255 \\
\hline A. 2 & 252 & 285 & 531 & 252 & 262 & 253 & 253 \\
\hline A. 3 & 232 & 270 & 473 & 232 & 243 & 232 & 235 \\
\hline A. 4 & 234 & 278 & 375 & 234 & 240 & 234 & 234 \\
\hline A. 5 & 236 & 272 & 349 & 236 & 240 & 236 & 236 \\
\hline B. 1 & 69 & 75 & 196 & 69 & 72 & 69 & 73 \\
\hline B. 2 & 76 & 87 & 243 & 76 & 79 & 76 & 79 \\
\hline B. 3 & 80 & 89 & 207 & 80 & 84 & 80 & 84 \\
\hline C. 1 & 227 & 261 & 442 & 227 & 237 & 227 & 229 \\
\hline C. 2 & 219 & 260 & 484 & 219 & 230 & 221 & 221 \\
\hline C. 3 & 243 & 268 & 551 & 243 & 249 & 244 & 245 \\
\hline C. 4 & 219 & 259 & 523 & 219 & 229 & 219 & 221 \\
\hline D. 1 & 60 & 72 & 184 & 60 & 64 & 60 & 61 \\
\hline D. 2 & 66 & 74 & 209 & 66 & 68 & 66 & 68 \\
\hline D. 3 & 72 & 83 & 221 & 72 & 77 & 72 & 74 \\
\hline
\end{tabular}

TABLE III: Experimental Results for Unweighted SCP

\begin{tabular}{|c|c|c|c|c|c|c|c|}
\hline \multirow{2}{*}{$\begin{array}{l}\text { Instance } \\
\text { number }\end{array}$} & \multirow{2}{*}{$\begin{array}{l}\text { Optimal } \\
\text { Solution } \\
\end{array}$} & \multirow{2}{*}{$\begin{array}{c}\text { Greedy } \\
\text { Algorithm }\end{array}$} & \multirow{2}{*}{$\begin{array}{c}\text { LP } \\
\text { Rounding }\end{array}$} & \multirow{2}{*}{$\begin{array}{c}{[18]} \\
\text { (Tabu Search) }\end{array}$} & \multirow{2}{*}{$\begin{array}{c}{[19]} \\
\text { Local Search for SCP }\end{array}$} & \multicolumn{2}{|c|}{ Proposed Algorithm } \\
\hline & & & & & & Start from Greedy & Start from LP Rounding \% \\
\hline 4.1 & 38 & 41 & 125 & 38 & 38 & 38 & 38 \\
\hline 4.10 & 38 & 43 & 127 & 38 & 38 & 39 & 39 \\
\hline 5.1 & 34 & 37 & 117 & 35 & 34 & 35 & 34 \\
\hline 6.1 & 21 & 23 & 107 & 21 & 21 & 21 & 23 \\
\hline 6.2 & 20 & 22 & 101 & 21 & 20 & 21 & 23 \\
\hline A. 1 & 39 & 42 & 186 & 39 & 39 & 40 & 47 \\
\hline A. 2 & 39 & 42 & 176 & 39 & 39 & 40 & 46 \\
\hline A. 3 & 39 & 43 & 172 & 39 & 39 & 40 & 44 \\
\hline A. 4 & 37 & 41 & 167 & 38 & 37 & 40 & 41 \\
\hline A. 5 & 38 & 43 & 170 & 38 & 38 & 40 & 43 \\
\hline B. 1 & 22 & 24 & 147 & 22 & 22 & 22 & 27 \\
\hline B. 2 & 22 & 23 & 154 & 22 & 22 & 22 & 25 \\
\hline B. 3 & 22 & 23 & 154 & 22 & 22 & 22 & 25 \\
\hline C. 1 & 40 & 47 & 214 & 43 & 43 & 40 & 49 \\
\hline C. 2 & 40 & 47 & 220 & 44 & 43 & 40 & 49 \\
\hline C. 3 & 40 & 47 & 163 & 43 & 43 & 40 & 45 \\
\hline C. 4 & 40 & 46 & 165 & 43 & 43 & 40 & 45 \\
\hline D. 1 & 25 & 27 & 216 & 25 & 25 & 25 & 39 \\
\hline D. 2 & 25 & 27 & 209 & 25 & 25 & 25 & 34 \\
\hline D. 3 & 24 & 27 & 206 & 25 & 24 & 24 & 33 \\
\hline
\end{tabular}

TABLE IV: Average quality ratio and GAP for the Weighted Set Covering Problem

\begin{tabular}{|c|c|c|}
\hline Algorithm & Average Quality Ratio & Average GAP \\
\hline Greedy Algorithm & 1.14 & 14.10 \\
Proposed (greedy initial solution) & 1.00 & 0.09 \\
LP Rounding & 2.22 & 122.57 \\
Proposed (LP initial solution) & 1.01 & 1.48 \\
\hline
\end{tabular}

TABLE V: Average quality ratio and GAP for the Unweighted Set Covering Problem

\begin{tabular}{|c|c|c|}
\hline Algorithm & Average Quality Ratio & Average GAP \\
\hline Greedy Algorithm & 1.11 & 10.66 \\
Proposed (greedy initial solution) & 1.02 & 1.58 \\
LP Rounding & 5.41 & 441.06 \\
Proposed (LP initial solution) & 1.18 & 17.6 \\
\hline
\end{tabular}

The average quality ratio and average GAP of LP rounding are 2.22 and 122.57 respectively for weighted SCP while for proposed approach they are 1.01 and 1.48. It is clearly visible that original greedy and LP Rounding are deviated from the optimal solution by a high degree where proposed approach hardly deviates from the optimal solution.

In Table V, the average quality ratio and average GAP of original greedy are 1.11 and 10.66 respectively for unweighted SCP while for proposed approach they are 1.02 and 1.58 . The average quality ratio and average GAP of LP rounding are 5.41 and 441.06 respectively for unweighted SCP while for proposed approach they are 1.18 and 17.6. It is clearly visible that original greedy and LP Rounding are highly deviated from the optimal solution where proposed approach hardly deviates from the optimal solution.

\section{COnClusion And Future Work}

This paper studies the existing approaches of MSCP and proposes a new heuristic approach for solving it. Appropriate theorems and algorithms are presented to clarify the proposed approach. The experimental results are compared with the existing results available in literature which shows the effectiveness of the proposed approach. This approach is tested only on OR-Libray in this work. In future this approach will be 
tested on some other libraries of SCP like Airline and bus scheduling problems and Railway scheduling problems. The proposed algorithm can also be tested in another popular NP hard problem called Vertex Cover Problem.

\section{ACKNOWLEDGEMENT}

The author would like to express her greatest gratitude to the anonymous reviewers for their constructive feedback and critical suggestions that helped significantly to elicit the utmost technical attribute of this research work.

\section{REFERENCES}

[1] G. Gens and E. Levner, "Complexity of approximation algorithms for combinatorial problems: a survey," ACM SIGACT News, vol. 12, no. 3, pp. 52-65, Fall 1980.

[2] R. M. Karp, "Reducibility among combinatorial problems," Springer US, pp. 85-103, March 1972.

[3] T. M. Chan, E. Grant, J. Knemann and M. Sharpe, "Weighted capacitated, priority, and geometric set cover via improved quasi-uniform sampling," Proceedings of the twenty-third annual ACM-SIAM symposium on Discrete Algorithms, pp. 1576-1585, SIAM, January 2012

[4] F. C. Gomes, C. N. Meneses, P. M. Pardalos and G. V. R. Viana, "Experimental analysis of approximation algorithms for the vertex cover and set covering problems," Computers and Operations Research, vol. 33, no. 12, pp. 3520-3534, December 2006.

[5] G. Lan, G. W. DePuy and G. E. Whitehouse, "An effctive and simple heuristic for the set covering problem," European Journal of Operational Research, vol. 176, no. 3, pp. 1387-1403, February 2007.

[6] L. Ruan, H. Du, X. Jia, W. Wu, Y. Li and K.-I Ko "A greedy approximation for minimum connected dominating sets," Theoretical Computer Science, vol. 329, no. 1, pp. 325-330, December 2004.

[7] N. Bilal, P. Galinier and F. Guibault, "A New Formulation of the Set Covering Problem for Metaheuristic Approaches," International Scholarly Research Notices, pp.1-10, April 2013.

[8] Y. Emek and A. Rosen, "Semi-streaming set cover," Automata, Languages, and Programming, pp. 453-464, Springer Berlin Heidelberg, July 2014.

[9] G. Lan, G. W. DePuy and G. E. Whitehouse, "An effective and simple heuristic for the set covering problem," European journal of operational research, vol. 176, no. 3, pp. 1387-1403, February 2007.

[10] F. Grandoni, A.Gupta, , S. Leonardi, P. Miettinen, P. Sankowski and M. Singh, "Set covering with our eyes closed," SIAM Journal on Computing, vol. 42, no. 3, pp. 808-830, May 2013.

[11] A. Deshpande, L. Hellerstein and D. Kletenik, "Approximation algorithms for stochastic boolean function evaluation and stochastic submodular set cover," Proceedings of the Twenty-Fifth Annual ACMSIAM Symposium on Discrete Algorithms, pp. 1453-1467, SIAM, January 2014.

[12] F. A. Chudak, M. X. Goemans, D. S. Hochbaum and D. P. Williamson, "A primaldual interpretation of two 2-approximation algorithms for the feedback vertex set problem in undirected graphs," Operations Research Letters, vol. 22, no. 4, pp. 111-118, May 1998.

[13] F. Colombo, R. Cordone and G. Lulli, "A variable neighborhood search algorithm for the multimode set covering problem," Journal of Global Optimization, pp. 1-20, August 2013.

[14] V. Chvatal, "A greedy heuristic for the set-covering problem," Mathematics of operations research, vol. 4, no. 3, pp. 233-235, August 1979.

[15] B. Saha and S. Khuller, "Set cover revisited: Hypergraph cover with hard capacities," Automata, Languages, and Programming, pp. 762773, Springer Berlin Heidelberg, July 2012.

[16] K. J. Lang, "Hill climbing beats genetic search on a boolean circuit synthesis problem of koza's," Proceedings of the Twelfth International Conference on Machine Learning, pp. 340-343, June 2014.

[17] J. E. Beasley, "OR-library: distributing test problems by electronic mail," Journal of the Operational Research Society, vol. 41, no. 11, pp. 1069-1072, November 1990.
[18] G. Kinney, J. W. Barnes and B. Colleti, "A group theoretic tabu search algorithm for set covering problems," Working paper, online available at http://www.me.utexas.edu/barnes/research/, 2004.

[19] N. Musliu, "Local search algorithm for unicost set covering problem," Springer Berlin Heidelberg, pp. 302-311, June 2006.

[20] B. Yelbay, S. I. Birbil and K. Bulbul, "The set covering problem revisited: an empirical study of the value of dual information," European Journal of Operational Research, September 2012. 